\title{
Catenulispora subtropica sp. nov. and Catenulispora yoronensis sp. nov.
}

\author{
Tomohiko Tamura, Yuumi Ishida, Misa Otoguro and Ken-ichiro Suzuki \\ NITE Biological Resource Center (NBRC), National Institute of Technology and Evaluation, 2-5-8 \\ Kazusakamatari, Kisarazu, Chiba 292-0818, Japan
}

Correspondence

Tomohiko Tamura

tamura-tomohiko@nite.go.jp
The genus Catenulispora (Busti et al., 2006) in the suborder Catenulisporineae (Cavaletti et al., 2006) has been well characterized based on acidophilic properties and chemotaxonomic features as well as on 16S rRNA gene-based phylogenetic analysis. The genus currently contains two species with validly published names, Catenulispora acidiphila (Busti et al., 2006) and Catenulispora rubra (Tamura et al., 2007).

In the course of research on the diversity of actinomycetes in Japan, three actinomycete strains were isolated from paddy-field soil samples and a forest soil sample. The strains contained LL-diaminopimelic acid ( $\left.\mathrm{LL}_{-} \mathrm{A}_{2} \mathrm{pm}\right)$ and glycine in the peptidoglycan, and phylogenetically belonged to the genus Catenulispora. Based on the data from a polyphasic taxonomic study, we propose that the three strains represent two novel species of the genus Catenulispora.

Strains TT $99-48^{\mathrm{T}}$ and TT 01-19 were isolated from paddyfield soil from Iriomote Island, Okinawa, in 1999, and Ishigaki Island, Okinawa, in 2001, respectively; strain TT $\mathrm{N} 02-20^{\mathrm{T}}$ was isolated from forest soil in Yoro Valley,

Abbreviation: LL-A 2 pm, LL-diaminopimelic acid.

The GenBank/EMBL/DDBJ accession numbers for the 165 rRNA gene sequences of strains TT $99-48^{\top}$ and TT NO2-20 ${ }^{\top}$ are AB327248 and AB327250, respectively.

A table showing the cellular fatty acid profiles of strains TT 99-48 ${ }^{\top}$ and TT N02-20 ${ }^{\top}$ and the type strains of recognized Catenulispora species is available as supplementary material with the online version of this paper.
Chiba, in 2002. The strains were all isolated using the yeast extract-SDS method (Hayakawa \& Nonomura, 1989) on HV medium (Hayakawa \& Nonomura, 1987) containing $20 \mathrm{mg}$ nalidixic acid $\mathrm{l}^{-1}$ and $50 \mathrm{mg}$ cycloheximide $\mathrm{l}^{-1}$.

PCR amplification, sequencing of the $16 \mathrm{~S}$ rRNA genes and phylogenetic analysis were performed as described previously (Tamura \& Hatano, 2001). Distances (distance options according to the Kimura two-parameter model) were calculated, and clustering was performed using the neighbour-joining (Saitou \& Nei, 1987) and maximumlikelihood (Felsenstein, 1981) methods; bootstrap values (Felsenstein, 1985) based on 1000 replications were used for this purpose. The 16S rRNA gene analysis showed that strains TT $99-48^{\mathrm{T}}$, TT $01-19$ and TT N02- $20^{\mathrm{T}}$ formed a monophyletic cluster with recognized members of the genus Catenulispora (Fig. 1). The $16 \mathrm{~S}$ rRNA gene sequence of strain TT $99-48^{\mathrm{T}}$ was identical to that of strain TT 01-19. The binary similarity values of the 16S rRNA gene sequences among strains TT $99-48^{\mathrm{T}}$, TT N02-20 ${ }^{\mathrm{T}}, C$. acidiphila NBRC $102108^{\mathrm{T}}$ and C. rubra Aac- $30^{\mathrm{T}}$ ranged from 97.4 to $98.7 \%$.

Cell-wall amino acids, the acyl type of peptidoglycan, whole-cell sugars, cellular fatty acids, mycolic acids, menaquinones and DNA base composition were analysed as described previously (Tamura et al., 1994). Freeze-dried cells for chemotaxonomic analyses were prepared from cultures grown in yeast extract-glucose broth $(1.0 \%$ yeast extract, $1.0 \%$ D-glucose, $\mathrm{pH} 7.2)$ on a rotary shaker at $28{ }^{\circ} \mathrm{C}$. The predominant menaquinones were MK-9 $\left(\mathrm{H}_{8}\right)$ 


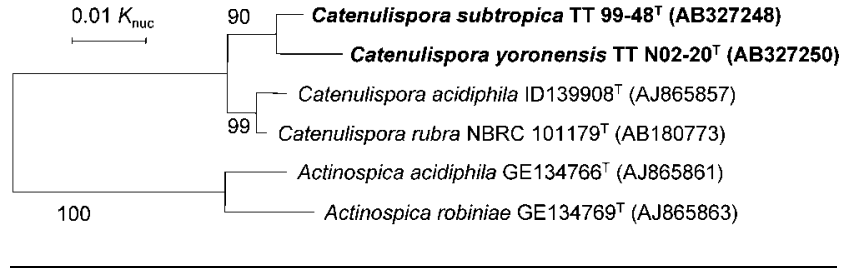

Fig. 1. Phylogenetic tree based on $16 \mathrm{~S}$ rRNA gene sequences of strains TT $99-48^{\top}$ and TT N02-20 ${ }^{\top}$ and members of the suborder Catenulisporineae. The tree was constructed using the neighbourjoining method. The $16 \mathrm{~S}$ rRNA gene sequences of Actinospica acidiphila GE134766 ${ }^{\top}$ (AJ865861) and Actinospica robiniae GE134769 ${ }^{\top}$ (AJ865863) were used as an outgroup. Bar, 0.01 $K_{\text {nuc }}$. Numbers at branch points are confidence limits (expressed as percentages) estimated from a bootstrap analysis of 1000 replicates.

and MK-9 $\left(\mathrm{H}_{6}\right)$ with MK-9 $\left(\mathrm{H}_{4}\right)$ and MK-9 $\left(\mathrm{H}_{10}\right)$ as minor components. The three strains contained LL- $\mathrm{A}_{2} \mathrm{pm}$, glycine, alanine and glutamic acid in the cell-wall, and mannose, arabinose and galactose as whole-cell sugars. The major cellular fatty acids of strains TT $99-48^{\mathrm{T}}$, TT $01-19$ and TT $\mathrm{N} 02-20^{\mathrm{T}}$ were iso- $\mathrm{C}_{16: 0}(46-63 \%)$ and anteiso- $\mathrm{C}_{17: 0}(14-$ $28 \%$ ) (see Supplementary Table S1, available in IJSEM online). Diphosphatidylglycerol was detected, whereas phosphatidylcholine, phosphatidylethanolamine and some other phospholipids containing glucosamine were not detected (phospholipid type PI sensu; Lechevalier et al., 1977). The DNA G+C contents were $69-71 \mathrm{~mol} \%$. Mycolic acids were not present. The acyl type of the cellwall muramic acid was acetyl type.

Morphological characteristics were observed using scanning electron microscopy as described previously (Tamura et al., 1994). Strains TT $99-48^{\mathrm{T}}$, TT $01-19$ and TT N02-20 formed rectiflexible or straight spore chains comprising rod-shaped spores, $0.5-0.8 \mu \mathrm{m}$ in diameter and $0.8-1.2 \mu \mathrm{m}$ in length. Determination of the cultural and physiological characteristics was performed as described by Seino et al. (1985) and Shirling \& Gottlieb (1966) and by using API ZYM, API $50 \mathrm{CH}$ and API Coryne (bioMérieux). The $\mathrm{pH}$ for growth was analysed by using filter-sterilized, yeast extract-glucose broth adjusted to various $\mathrm{pH}$ values with sulfuric acid. Detailed results of these analyses are provided in the species description and in Tables 1 and 2. Strains TT 99- $48^{\mathrm{T}}$ and TT $01-19$ shared very similar characteristics, but strain TT N02-20 ${ }^{\mathrm{T}}$ showed obvious differences. They all grew optimally at $\mathrm{pH} 6-7$; strains TT $99-48^{\mathrm{T}}$ and TT $01-$ 19 grew at $\mathrm{pH} \mathrm{8,}$ but strain TT N02-20 ${ }^{\mathrm{T}}$ did not. The physiological characteristics of these three strains were clearly different from those of C. acidiphila NBRC $102108^{\mathrm{T}}$ and C. rubra Aac $-30^{\mathrm{T}}$ (Table 2).

The microplate hybridization method developed by Ezaki et al. $(1988,1989)$ was used to determine DNA-DNA relatedness. The DNA-DNA relatedness values between

Table 1. Cultural characteristics of strains TT $99-48^{\top}$ (Catenulispora subtropica sp. nov.) and TT N02-20 ${ }^{\top}$ (Catenulispora yoronensis sp. nov.)

Colour codes in parentheses correspond to those given by Rayner (1970). The diffusible pigment was not produced in all the media tested without tyrosine agar. -, Negative for diffusible pigment.

\begin{tabular}{|c|c|c|}
\hline Characteristic & Strain TT $99-48^{\mathrm{T}}$ & Strain TT N02-20 ${ }^{\mathrm{T}}$ \\
\hline Growth & Moderate & Good \\
\hline \multicolumn{3}{|l|}{ Oatmeal agar (ISP 3) } \\
\hline Growth & Moderate & Good \\
\hline \multicolumn{3}{|c|}{ Inorganic salts-starch agar (ISP 4) } \\
\hline Growth & Poor & Good \\
\hline Colour of colony & Pale yellow (45) & Pale yellow (45) \\
\hline \multicolumn{3}{|c|}{ Glycerol-asparagine agar (ISP 5) } \\
\hline Growth & Poor & Poor \\
\hline \multicolumn{3}{|l|}{ Tyrosine agar (ISP 7) } \\
\hline Growth & Moderate & Poor \\
\hline Colour of colony & Greyish-white & Greyish-white \\
\hline Diffusible pigment & Greyish reddish-orange (59) & - \\
\hline
\end{tabular}


Table 2. Differential characteristics of strains TT $99-48^{\top}$ and TT 01-19 (Catenulispora subtropica sp. nov.), TT N02-20 ${ }^{\top}$ (Catenulispora yoronensis sp. nov.) and recognized species of the genus Catenulispora

Strains: 1 , TT 99-48 ${ }^{\mathrm{T}} ; 2$, TT 01-19; 3, TT N02-20 $; 4$, C. acidiphila NBRC $102108^{\mathrm{T}}$; 5, C. rubra Aac- $30^{\mathrm{T}}$. All strains utilize D-xylose, Dglucose, D-mannose, arbutin, aesculin, ferric citrate, D-cellobiose, maltose, trehalose, starch, glycogen, gentiobiose and L-fucose, but not erythritol, D-arabinose, D-ribose, L-xylose, D-adonitol, L-sorbose, dulcitol, inositol, D-sorbitol, methyl $\alpha$-D-glucopyranoside, inulin, Dmelezitose, xylitol, D-lyxose, D-tagatose, D-fucose, D-arabitol, Larabitol, 2-ketogluconate or 5-ketogluconate. All strains are positive for pyrazinamidase, alkaline phosphatase, $\alpha$-glucosidase, $N$-acetyl- $\beta$ glucosaminidase, aesculin hydrolysis, leucine aminopeptidase, valine aminopeptidase, acid phosphatase, phosphohydrolase, $\beta$-glucosidase and $\alpha$-mannosidase; and negative for pyrrolidonyl arylamidase, urea hydrolysis, lipase, cystine aminopeptidase and $\alpha$-galactosidase. + , Positive; -, negative; w, weakly positive.

\begin{tabular}{|c|c|c|c|c|c|}
\hline Characteristic & 1 & 2 & 3 & 4 & 5 \\
\hline Nitrate reduction & + & + & - & - & - \\
\hline$\beta$-Glucuronidase & - & - & - & - & + \\
\hline$\beta$-Galactosidase & + & + & - & + & $\mathrm{W}$ \\
\hline Gelatin hydrolysis & + & + & - & + & - \\
\hline Catalase & + & + & + & + & - \\
\hline Trypsin & + & + & - & - & + \\
\hline$\alpha$-Fucosidase & + & + & + & - & - \\
\hline \multicolumn{6}{|l|}{ Utilization of: } \\
\hline Glycerol & + & + & - & - & + \\
\hline L-Arabinose & + & + & - & - & + \\
\hline Methyl $\beta$-D-xylopyranoside & w & + & - & - & - \\
\hline D-Galactose & + & + & - & + & - \\
\hline D-Fructose & - & - & - & + & + \\
\hline D-Mannitol & - & - & - & - & + \\
\hline Methyl $\alpha$-D-glucopyranoside & - & - & - & - & + \\
\hline $\mathrm{N}$-Acetylglucosamine & + & + & - & $\mathrm{w}$ & + \\
\hline Salicin & + & + & - & + & $\mathrm{w}$ \\
\hline Sucrose & - & - & $\mathrm{W}$ & - & + \\
\hline Gluconate & + & + & + & + & - \\
\hline $\mathrm{pH}$ for growth & $5-8$ & $5-8$ & $5-7$ & $5-8$ & $4-6.5$ \\
\hline
\end{tabular}

strain TT 99- $48^{\mathrm{T}}$ and strain TT N02-20 $0^{\mathrm{T}}$, C. rubra Aac- $30^{\mathrm{T}}$ and C. acidiphila NBRC $102108^{\mathrm{T}}$ were below $36 \%$; however, the level of relatedness with strain TT 01-19 was $74 \%$. The DNA-DNA relatedness between strain TT N02- $20^{\mathrm{T}}$ and strains TT $99-48^{\mathrm{T}}$ and TT $01-19$ and C. rubra Aac- $30^{\mathrm{T}}$ and C. acidiphila NBRC $102108^{\mathrm{T}}$ was below $36 \%$. Strain TT 01-19 was identified as representing the same species as TT $99-48^{\mathrm{T}}$. Strains TT $99-48^{\mathrm{T}}$ and TT N02-20 could also be distinguished from recognized Catenulispora species based on several phenotypic properties (Table 2). Therefore, based on the morphological, physiological and chemotaxonomic data, DNA-DNA relatedness and phylogenetic distinctiveness, the three strains should be assigned as representing two novel species for which the names Catenulispora subtropica sp. nov. and Catenulispora yoronensis sp. nov. are proposed.
The two recognized species of the genus Catenulispora, $C$. acidiphila and C. rubra are acidophilic. Consequently, $C$. acidiphila was characterized as an organism that possessed glucose, xylose, ribose, rhamnose and arabinose as wholecell sugars in the genus description (Busti et al., 2006). Conversely, the novel isolates are non-acidophilic and possess mannose, arabinose and galactose. These facts justify an emendation of the description of the genus Catenulispora.

\section{Emended description of the genus Catenulispora Busti et al. 2006}

Catenulispora (Ca.te.nu.li.spo'ra. L. fem. n. catenula small chain; Gr. fem. n. spora seed; N.L. fem. n. Catenulispora a thin chain of spores).

Gram-positive, acidophilic or non-acidophilic, non-acidfast, aerobic organisms that form branching hyphae. Nonfragmentary vegetative mycelium and aerial hyphae starting to septate in chains of cylindrical arthrospores are produced. Motile elements are not produced. Peptidoglycan contains $\mathrm{LL}_{-} \mathrm{A}_{2} \mathrm{pm}$, glycine, glutamic acid and alanine. Mannose, arabinose and galactose are detected as whole-cell sugars; xylose, rhamnose, ribose and glucose are also detected. Iso- $\mathrm{C}_{16: 0}$ and anteiso- $\mathrm{C}_{17: 0}$ are present as major cellular fatty acids. Menaquinones MK- $9\left(\mathrm{H}_{6}\right)$, MK$9\left(\mathrm{H}_{4}\right)$ and MK- $9\left(\mathrm{H}_{8}\right)$ are predominant. Diphosphatidylglycerol, phosphatidylinositol and phosphatidylinositol mannosides are present, whereas phosphatidylethanolamine and phosphatidylcholine are absent. The $\mathrm{G}+\mathrm{C}$ content of the DNA is $69-72 \mathrm{~mol} \%$. The type species is Catenulispora acidiphila. Phylogenetically, the genus Catenulispora is a member of the family Catenulisporaceae within the suborder Catenulisporineae of the order Actinomycetales.

\section{Description of Catenulispora subtropica sp. nov.}

Catenulispora subtropica (sub.tro.pi'ca. N.L. fem. adj. subtropica pertaining to subtropical zone, the origin of the soil sample from which the type strain was isolated).

Gram-positive, aerobic actinomycete that forms extensively branched substrate hyphae. Vegetative hyphae are finely branched and do not fragment. Monopodially or dichotomously branching aerial mycelia develop sparsely. Greyish reddish-orange colonies are formed on ISP 2. Temperature range for growth is $10-37^{\circ} \mathrm{C}$, with optimum growth at $25-$ $30{ }^{\circ} \mathrm{C}$. $\mathrm{pH}$ range for growth is 5 or 6 to 8 , with optimum growth at pH 6-7. Glycerol, L-arabinose, D-xylose, methyl $\beta$ D-xylopyranoside, D-galactose, D-glucose, D-mannose, $\mathrm{N}$ acetylglucosamine, arbutin, aesculin, ferric citrate, salicin, Dcellobiose, maltose, starch, glycogen, gentiobiose, L-fucose and gluconate are used as sole carbon sources. Positive for catalase, alkaline phosphatase, nitrate reduction, pyrazinamidase, leucine aminopeptidase, valine aminopeptidase, trypsin, acid phosphatase, phosphohydrolase, $\beta$-galactosidase, $\alpha$-glucosidase, $\beta$-glucosidase, $N$-acetyl- $\beta$-glucosamini- 
dase, $\alpha$-mannosidase and $\alpha$-fucosidase; negative for pyrrolidonyl arylamidase, esterase (C4), esterase lipase (C8), lipase (C14), cystine aminopeptidase, chymotrypsin, $\alpha-$ galactosidase and $\beta$-glucuronidase. Aesculin and gelatin are hydrolysed, but not urea. Predominant menaquinones are MK-9 $\left(\mathrm{H}_{8}\right)$ and MK-9(- $\left.\mathrm{H}_{6}\right)$. Predominant cellular fatty acids are iso- $\mathrm{C}_{16: 0}$ and anteiso- $\mathrm{C}_{17: 0}$. Cell wall contains LL$\mathrm{A}_{2} \mathrm{pm}$, glycine, glutamic acid and alanine (peptidoglycan type A3 $\gamma$ sensu; Schleifer \& Kandler, 1972). Contains mannose, arabinose and galactose as whole-cell sugars. The DNA G $+\mathrm{C}$ content is $70-71 \mathrm{~mol} \%$.

The type strain, TT $99-48^{\mathrm{T}}\left(=\mathrm{NBRC} 103395^{\mathrm{T}}=\mathrm{KCTC}\right.$ $\left.19328^{\mathrm{T}}\right)$, was isolated from a paddy rice soil from Iriomote Island, Japan. Strain TT 01-19 (=NBRC 103396) is a reference strain.

\section{Description of Catenulispora yoronensis sp. nov.}

Catenulispora yoronensis (yo.ro.nen'sis. N.L. fem. adj. yoronensis pertaining to Yoro Valley, Chiba, Japan, the origin of the soil sample from which the type strain was isolated).

Gram-positive, aerobic actinomycete that forms extensively branched substrate hyphae. Vegetative hyphae are finely branched and do not fragment. Monopodially or dichotomously branching aerial mycelia develop sparsely. Light to moderate yellowish brown and/or moderate yellow colonies are formed on ISP 2. Temperature range for growth is 10 $37^{\circ} \mathrm{C}$, with optimum growth at $25-30{ }^{\circ} \mathrm{C}$. $\mathrm{pH}$ range for growth is $5-7$, with optimum growth at $\mathrm{pH}$ 6-7. D-Xylose, D-glucose, D-mannose, arbutin, aesculin, ferric citrate, Dcellobiose, maltose, D-melibiose, trehalose, starch, glycogen, L-fucose and gluconate are used as sole carbon sources. Positive for catalase, pyrazinamidase, alkaline phosphatase, leucine aminopeptidase, valine aminopeptidase, acid phosphatase, phosphohydrolase, $\alpha$-glucosidase, $\beta$-glucosidase, $N$ acetyl- $\beta$-glucosaminidase, $\alpha$-mannosidase and $\alpha$-fucosidase; negative for nitrate reduction, pyrrolidonyl arylamidase, esterase (C4), esterase lipase (C8), lipase (C14), cystine aminopeptidase, $\alpha$-galactosidase, trypsin, chymotrypsin, $\beta$ galactosidase and $\beta$-glucuronidase. Aesculin is hydrolysed, but gelatin and urea are not. Predominant menaquinones are MK-9 $\left(\mathrm{H}_{8}\right)$ and MK-9 $\left(\mathrm{H}_{6}\right)$. Predominant cellular fatty acids are iso- $\mathrm{C}_{16: 0}$ and anteiso- $\mathrm{C}_{17: 0}$. Cell wall contains LL$\mathrm{A}_{2} \mathrm{pm}$, glycine, glutamic acid and alanine (peptidoglycan type A3 $\gamma$ sensu; Schleifer \& Kandler, 1972). Contains mannose, arabinose and galactose as whole-cell sugars. The DNA G + C content of the type strain is $69 \mathrm{~mol} \%$.

The type strain, TT N02-20 $\left(=\right.$ NBRC $103397^{\mathrm{T}}=$ KCTC $19327^{\mathrm{T}}$ ), was isolated from forest soil from Yoro Valley, Japan.

\section{Acknowledgements}

This work was supported by a Grant-in-Aid for Scientific Research (C) (2) No. 11660326 from the Japan Society for the Promotion of Science. The authors are grateful to Dr Akira Nakagiri for kind support for sampling. We wish to thank Drs Stefano Donadio and Linda Cavaletti for valuable comments and advice.

\section{References}

Busti, E., Cavaletti, L., Monciardini, P., Schumann, P., Rohde, M., Sosio, M. \& Donadio, S. (2006). Catenulispora acidiphila gen. nov., sp. nov., a novel, mycelium-forming actinomycete, and proposal of Catenulisporaceae fam. nov. Int J Syst Evol Microbiol 56, 1741-1746.

Cavaletti, L., Monciardini, P., Schumann, P., Rohde, M., Bamonte, R., Busti, E., Sosio, M. \& Donadio, S. (2006). Actinospica robiniae gen. nov., sp. nov. and Actinospica acidiphila sp. nov.: proposal for Actinospicaceae fam. nov. and Catenulisporinae subord. nov. in the order Actinomycetales. Int J Syst Evol Microbiol 56, 1747-1753.

Ezaki, T., Hashimoto, Y., Takeuchi, N., Yamamoto, H., Liu, S.-L., Miura, H., Matsui, K. \& Yabuuchi, E. (1988). Simple genetic identification method of viridans group streptococci by colorimetric dot hybridization and quantitative fluorometric hybridization in microdilution wells. J Clin Microbiol 26, 1708-1713.

Ezaki, T., Hashimoto, Y. \& Yabuuchi, E. (1989). Fluorometric deoxyribonucleic acid-deoxyribonucleic acid hybridization in microdilution wells as an alternative to membrane filter hybridization in which radioisotopes are used to determine genetic relatedness among bacterial strains. Int J Syst Bacteriol 39, 224-229.

Felsenstein, J. (1981). Evolutionary trees from DNA sequences: a maximum likelihood approach. J Mol Evol 17, 368-376.

Felsenstein, J. (1985). Confidence limits on phylogenies: an approach using the bootstrap. Evolution 39, 783-791.

Hayakawa, M. \& Nonomura, H. (1987). Humic acid-vitamin agar, a new medium for selective isolation of soil actinomycetes. J Ferment Technol 65, 501-509.

Hayakawa, M. \& Nonomura, H. (1989). A new method for the intensive isolation of actinomycetes from soil. Actinomycetol 3, 95104.

Lechevalier, M. P., DeBièvre, C. \& Lechevalier, H. A. (1977). Chemotaxonomy of aerobic actinomycetes: phospholipid composition. Biochem Syst Ecol 5, 249-260.

Rayner, R. W. (1970). A Mycological Colour Chart: Kew, UK: Commonwealth Mycological Institute and British Mycological Society.

Saitou, N. \& Nei, M. (1987). The neighbor-joining method: a new method for reconstructing phylogenetic trees. Mol Biol Evol 4, 406425.

Schleifer, K. H. \& Kandler, O. (1972). Peptidoglycan types of bacterial cell walls and their taxonomic implications. Bacteriol Rev 36, 407-477.

Seino, A., Arai, M., Enokita, R., Okazaki, T. \& Furuichi, A. (1985). Identification Manual of Actinomycetes. Tokyo: The Society for Actinomycetes Japan (in Japanese).

Shirling, E. B. \& Gottlieb, D. (1966). Methods for characterization of Streptomyces species. Int J Syst Bacteriol 16, 313-340.

Tamura, T. \& Hatano, K. (2001). Phylogenetic analysis of the genus Actinoplanes and transfer of Actinoplanes minutisporangius Ruan et al. 1986 and 'Actinoplanes aurantiacus' to Cryptosporangium minutisporangium comb. nov. and Cryptosporangium aurantiacum sp. nov. Int $J$ Syst Evol Microbiol 51, 2119-2125.

Tamura, T., Nakagaito, Y., Nishii, T., Hasegawa, T., Stackebrandt, E. \& Yokota, A. (1994). A new genus of the order Actinomycetales, Couchioplanes gen. nov., with descriptions of Couchioplanes caeruleus (Horan and Brodsky 1986) comb. nov. and Couchioplanes caeruleus subsp. azureus subsp. nov. Int J Syst Bacteriol 44, 193-203.

Tamura, T., Ishida, Y., Sakane, T. \& Suzuki, K. (2007). Catenulispora rubra sp. nov., an acidophilic actinomycete isolated from forest soil. Int J Syst Evol Microbiol 57, 2272-2274. 